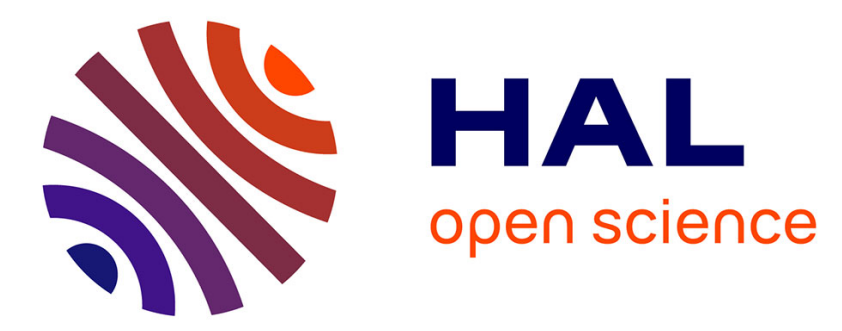

\title{
Optimal control of slender microswimmers
}

Marta Zoppello, Antonio Desimone, François Alouges, Laetitia Giraldi, Pierre

Martinon

\section{To cite this version:}

Marta Zoppello, Antonio Desimone, François Alouges, Laetitia Giraldi, Pierre Martinon. Optimal control of slender microswimmers. Gerisch, Alf and Penta, Raimondo and Lang, Jens. Multiscale Models in Mechano and Tumor Biology, Springer International Publishing, pp.21, 2017, 978-3-31973371-5. 10.1007/978-3-319-73371-5_8. hal-01393327

\section{HAL Id: hal-01393327 https://hal.science/hal-01393327}

Submitted on 9 Nov 2016

HAL is a multi-disciplinary open access archive for the deposit and dissemination of scientific research documents, whether they are published or not. The documents may come from teaching and research institutions in France or abroad, or from public or private research centers.
L'archive ouverte pluridisciplinaire HAL, est destinée au dépôt et à la diffusion de documents scientifiques de niveau recherche, publiés ou non, émanant des établissements d'enseignement et de recherche français ou étrangers, des laboratoires publics ou privés. 


\section{Contents}

1 Optimal control of slender microswimmers .............. 1

M. Zoppello, A. DeSimone, F. Alouges, L. Giraldi, P. Martinon

1.1 Mathematical setting of the problem $\ldots \ldots \ldots \ldots \ldots \ldots \ldots .2$

1.1.1 Kinematics of the N-link swimmer ............. 2

1.1.2 Equations of motion ..................... 3

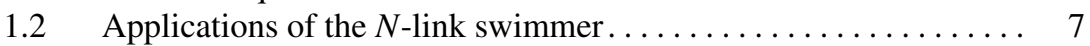

1.2.1 Curvature approximation $\ldots \ldots \ldots \ldots \ldots \ldots \ldots \ldots .6$

1.2.2 $N$-link approximation of sperm cell swimmer ........ 9

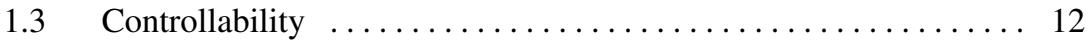

1.3.1 Classical results in geometric control .............. 13

1.3.2 Regularity............................. 14

1.3.3 Controllability of the Purcell Swimmer $(N=3) \ldots \ldots \ldots .14$

1.3.4 Controllability of the $N$-link swimmer . .......... 15

1.4 Minimum time optimal control problem for the $N$-link swimmer . . 16

1.4.1 Minimum Time Problem ..................... 16

1.4.2 Numerical Optimization ....................... 17

1.5 Numerical simulations for the Purcell's swimmer $(\mathrm{N}=3) \ldots \ldots 17$

1.5.1 The classical Purcell stroke ................. 19

1.5.2 Comparison of the optimal stroke and Purcell stroke ... 19

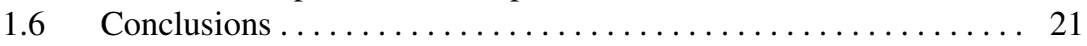

References $\ldots \ldots \ldots \ldots \ldots \ldots \ldots \ldots \ldots \ldots \ldots \ldots \ldots \ldots \ldots \ldots \ldots \ldots \ldots \ldots, 22$

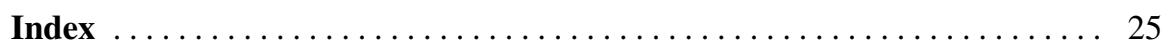





\title{
List of Contributors
}

\author{
Marta Zoppello \\ Universitá degli studi di Trento, Via sommarive 14 Povo (TN) Italy, e-mail: \\ marta.zoppello@unitn.it \\ Antonio DeSimone \\ Scuola Internazionale di Studi Superiori Avanzati (SISSA) via Bonomea 265 \\ Trieste Italy e-mail: desimone@ sissa.it \\ François Alouges \\ École Polytechnique CNRS, Route de Saclay, 91128 Palaiseau France, e-mail: \\ francois.alouges@polytechnique.edu \\ Laetitia Giraldi \\ INRIA Sophia Antipolis Méditerranée , e-mail: laetitia.giraldi@inria.fr \\ Pierre Martinon \\ Team COMMANDS, INRIA Saclay - CMAP Polytechnique France, e-mail: \\ pierre.martinon@inria.fr
}





\section{Acronyms}

RTF Resistive Force Theory

OCP Optimal Control Problem

NLP NonLinear Programming 



\title{
Chapter 1 Optimal control of slender microswimmers
}

\author{
M. Zoppello, A. DeSimone, F. Alouges, L. Giraldi, P. Martinon
}

\begin{abstract}
We discuss a reduced model to compute the motion of slender swimmers which propel themselves by changing the curvature of their body. Our approach is based on the use of Resistive Force Theory for the evaluation of the viscous forces and torques exerted by the surrounding fluid, and on discretizing the kinematics of the swimmer by representing its body through an articulated chain of $N$ rigid links capable of planar deformations. The resulting system of ODEs governing the motion of the swimmer is easy to assemble and to solve, making our reduced model a valuable tool in the design and optimization of bio-inspired artificial microdevices. We prove that the swimmer is controllable in the whole plane for $N \geq 3$ and for almost every set of stick lengths. As a direct result, there exists an optimal swimming strategy to reach a desired configuration in minimum time. Numerical experiments for $N=3$ (Purcell swimmer) suggest that the optimal strategy is periodic, namely a sequence of identical strokes. Our results indicate that this candidate for an optimal stroke indeed gives a better displacement speed than the classical Purcell stroke.
\end{abstract}

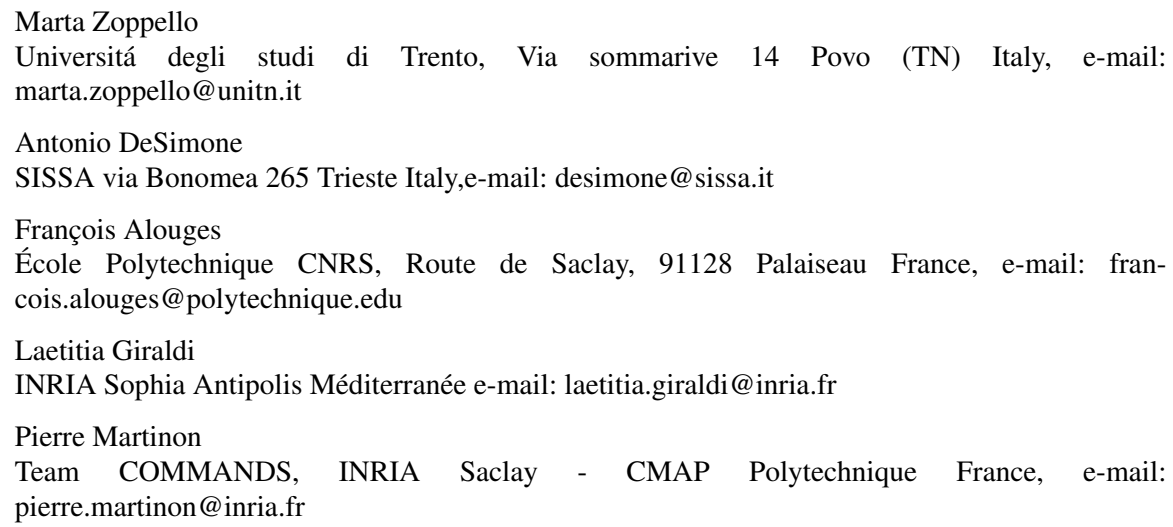




\subsection{Mathematical setting of the problem}

In this section we describe the kinematics of the so called $N$-link swimmer, inspired by the Purcell's 3-link swimmer. A discrete representation od the swimmer's curvature is provided by he angles between successive inks. These angles are considered as freely prescribed shape parameters. We then write the balance of total viscous force and torque, i.e. the equations of motion, solving for the time evolution of position and orientation of the swimmer in response to a prescribed history of (concentrated) curvatures along the swimmer's body.

\subsubsection{Kinematics of the N-link swimmer}

Here we are interested in essentially one-dimensional swimmers moving in a plane. This setting is suitable for the study of slender, one-dimensional swimmers exploring planar trajectories. The general case is a bit more involved because of the nonadditivity of three-dimensional rotations, see e.g. [1], but it can be handled with similar techniques.

Our swimmer is composed of $N$ rigid links with joints at their ends (see Fig. 1.1), moving in the plane $\left(\mathbf{e}_{x}, \mathbf{e}_{y}\right)$ (2d lab-frame). We set $\mathbf{e}_{z}:=\mathbf{e}_{x} \times \mathbf{e}_{y}$. The $i$-th link is the segment with end points $\mathbf{x}_{i}$ and $\mathbf{x}_{i+1}$. It has length $L_{i}>0$ and makes an angle $\theta_{i}$ with the vector $\mathbf{e}_{x}$. The lenght of the sticks is chosen such that the size of the swimmer is of order of $\mu \mathrm{m}$. We define by $\mathbf{x}_{i}:=\left(x_{i}, y_{i}\right)(i=1, \cdots, N)$ the coordinates of the first end of each link. Notice that, for $i \in\{2 \cdots N\}, \mathbf{x}_{i}$ is a function of $\mathbf{x}_{1}, \theta_{k}$ and $L_{k}$, with $k \in\{1 \cdots i-1\}$ :

$$
\mathbf{x}_{i}:=x_{1}+\sum_{k=1}^{i-1} L_{k}\left(\begin{array}{c}
\cos \left(\theta_{k}\right) \\
\sin \left(\theta_{k}\right)
\end{array}\right) .
$$

The swimmer is described by two kind of variables:

- the state variables which denote the position and the orientation of one selected link, labeled as the $i *$-th one;

- the shape variables which describe the relative angles between successive links. For each link with $i>i *$, this is the angle relative to the preceding one, denoted by $\alpha_{i}=\theta_{i}-\theta_{i-1}$, for $i *<i \leq N$. For $i<i *$ this is the the angle relative to the following one, denoted by $\alpha_{i}=\theta_{i+1}-\theta_{i}$, for $1 \leq i<i *$.

For example, if the triplet $\left(\mathbf{x}_{1}, \theta_{1}\right)$ is the state of the swimmer then the vector $\left(\alpha_{2}=\theta_{2}-\theta_{1}, \cdots, \alpha_{N}=\theta_{N}-\theta_{N-1}\right)$ describes the shape of the swimmer. We will use these coordinates in the rest of the chapter. 


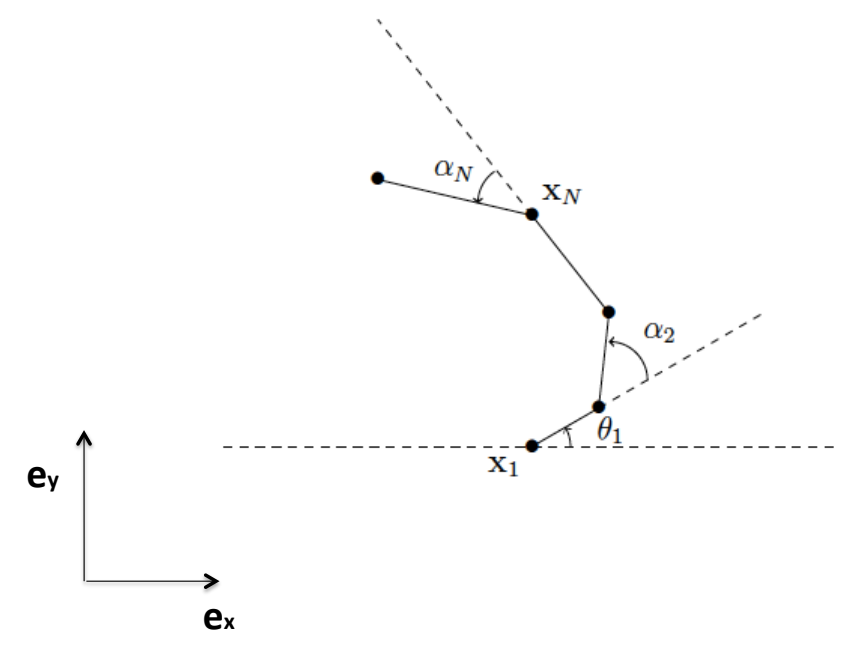

Fig. 1.1 Coordinates of the $N$-link swimmer

\subsubsection{Equations of motion}

The dynamics of the swimmer is governed by a system of three ODEs.

This system represents the Newton laws, in which inertia is neglected, namely

$$
\left\{\begin{array}{l}
\mathbf{F}=0 \\
\mathbf{e}_{z} \cdot \mathbf{T}_{\mathbf{x}_{1}}=0
\end{array}\right.
$$

where $\mathbf{F}$ is the total force that the fluid exerts on the swimmer and $\mathbf{T}_{\mathbf{x}_{1}}$ is the corresponding total torque computed with respect to the point $\mathbf{x}_{1}$.

In what follows we use the local drag approximation of Resistive Force Theory (RTF), to couple the fluid and the swimmer. According to this approximation the hydrodinamic forces are linear in the velocities of each point. More precisely denoting by $s$ the arc length coordinate on the $i$-th link $\left(0 \leq s \leq L_{i}\right)$, by $\mathbf{v}_{i}(s)$ the velocity of the corresponding point, and calling $\mathbf{e}_{i}=\left(\begin{array}{c}\cos \left(\theta_{i}\right) \\ \sin \left(\theta_{i}\right)\end{array}\right)$ and $\mathbf{e}_{i}^{\perp}=\left(\begin{array}{c}-\sin \left(\theta_{i}\right) \\ \cos \left(\theta_{i}\right)\end{array}\right)$ the unit vectors in the directions parallel and perpendicular to the $i$-th link respectively, we can write $\mathbf{x}_{i}(s)=\mathbf{x}_{i}+s \mathbf{e}_{i}$. Differentiating, we obtain, 


$$
\mathbf{v}_{i}(s)=\dot{\mathbf{x}}_{i}+s \dot{\theta}_{i} \mathbf{e}_{i}^{\perp} .
$$

According to RTF, the density of the force $\mathbf{f}_{i}$ acting on the $i$-th segment depends linearly on the velocity and can be written as

$$
\mathbf{f}_{i}(s):=-\xi\left(\mathbf{v}_{i}(s) \cdot \mathbf{e}_{i}\right) \mathbf{e}_{i}-\eta\left(\mathbf{v}_{i}(s) \cdot \mathbf{e}_{i}^{\perp}\right) \mathbf{e}_{i}^{\perp},
$$

where $\xi$ and $\eta$ are the drag coefficients in the directions of $\mathbf{e}_{i}$ and $\mathbf{e}_{i}^{\perp}$ respectively, measured in $\mathrm{Nsm}^{-2}$. We thus obtain

$$
\left\{\begin{array}{l}
\mathbf{F}=\sum_{i=1}^{N} \int_{0}^{L_{i}} \mathbf{f}_{i}(s) d s \\
\mathbf{e}_{z} \cdot \mathbf{T}_{\mathbf{x}_{1}}=\mathbf{e}_{z} \cdot \sum_{i=1}^{N} \int_{0}^{L_{i}}\left(\mathbf{x}_{i}(s)-\mathbf{x}_{1}\right) \times \mathbf{f}_{i}(s) d s .
\end{array}\right.
$$

Using (1.3) and (1.4) into (1.5), the total force and torque can be expressed as

$$
\mathbf{F}=-\sum_{i=1}^{N} L_{i} \xi\left(\dot{\mathbf{x}}_{i} \cdot \mathbf{e}_{i}\right) \mathbf{e}_{i}+\left(L_{i} \eta\left(\dot{\mathbf{x}}_{i} \cdot \mathbf{e}_{i}^{\perp}\right)+\frac{L_{i}^{2}}{2} \eta \dot{\theta}_{i}\right) \mathbf{e}_{i}^{\perp}
$$

and

$$
\begin{aligned}
\mathbf{e}_{z} \cdot \mathbf{T}_{\mathbf{x}_{1}} & =-\sum_{i=1}^{N} \frac{L_{i}^{2}}{2} \eta\left(\dot{\mathbf{x}}_{i} \cdot \mathbf{e}_{i}^{\perp}\right)+\frac{L_{i}^{3}}{3} \eta \dot{\theta}_{i} \\
& +\left(\mathbf{x}_{i}-\mathbf{x}_{1}\right) \times\left(L_{i} \xi\left(\dot{\mathbf{x}}_{i} \cdot \mathbf{e}_{i}\right) \mathbf{e}_{i}+\left(L_{i} \eta\left(\dot{\mathbf{x}}_{i} \cdot \mathbf{e}_{i}^{\perp}\right)+\frac{L_{i}^{2}}{2} \eta \dot{\theta}_{i}\right) \mathbf{e}_{i}^{\perp}\right) \cdot \mathbf{e}_{z} .
\end{aligned}
$$

Moreover, the differentiation of (1.1) gives

$$
\dot{\mathbf{x}}_{i}=\dot{\mathbf{x}}_{1}+\sum_{k=1}^{i-1} L_{k} \dot{\theta}_{k} \mathbf{e}_{k}^{\perp}
$$

which is linear in $\dot{\mathbf{x}}_{1}$ and $\left(\dot{\theta}_{k}\right)_{1 \leq k \leq N}$. This implies that also (1.6) and (1.7) are linear in $\dot{\mathbf{x}}_{1}$ and $\dot{\theta}_{i}$ for $i \in[1 \cdots N]$, and therefore system (1.2) reads

$$
\left(\begin{array}{c}
\mathbf{F} \\
\mathbf{e}_{z} \cdot \mathbf{T}_{\mathbf{x}_{1}}
\end{array}\right)=\mathbf{M}\left(\theta_{1}, \cdots, \theta_{N}\right)\left(\begin{array}{c}
\dot{\mathbf{x}}_{1} \\
\dot{\theta}_{1} \\
\dot{\theta}_{2} \\
\vdots \\
\dot{\theta}_{N}
\end{array}\right)=\left(\begin{array}{c}
0 \\
0 \\
0
\end{array}\right) .
$$

We point out that for all $i \in\{2, \cdots, N\}, \alpha_{i}=\theta_{i}-\theta_{i-1}$, equations (1.6) and (1.7) can be written using the relative angles $\left(\alpha_{i}\right)_{i=2, \cdots, N}$ instead of the variables $\left(\theta_{i}\right)_{2 \leq i \leq N}$ recalling that 


$$
\theta_{i}=\theta_{i-1}+\alpha_{i}, \quad i=2, \cdots, N .
$$

To this end, we introduce the matrix $\mathbf{C}$ defined by

$$
\mathbf{C}=\left(\begin{array}{ccccccc}
1 & 0 & \cdots & \cdots & \cdots & \cdots & 0 \\
0 & 1 & \ddots & \ddots & \ddots & \ddots & \vdots \\
0 & 0 & 1 & \ddots & \ddots & & \vdots \\
0 & 0 & -1 & \ddots & \ddots & & \vdots \\
\vdots & \vdots & 0 & \ddots & \ddots & & 0 \\
\vdots & \vdots & \vdots & \ddots & \ddots & \ddots & 0 \\
0 & 0 & 0 & \cdots & 0 & -1 & 1
\end{array}\right)
$$

and obtain

$$
\mathbf{C}\left(\begin{array}{c}
\dot{\mathbf{x}}_{1} \\
\dot{\theta}_{1} \\
\dot{\theta}_{2} \\
\vdots \\
\dot{\theta}_{N}
\end{array}\right)=\left(\begin{array}{c}
\dot{\mathbf{x}}_{1} \\
\dot{\theta}_{1} \\
\dot{\alpha}_{2} \\
\vdots \\
\dot{\alpha}_{N}
\end{array}\right)
$$

Thus, by setting

$$
\mathbf{N}\left(\theta_{1}, \alpha_{2}, \cdots, \alpha_{N}\right):=\mathbf{M}\left(\theta_{1}, \theta_{2}\left(\theta_{1}, \alpha_{2}, \cdots, \alpha_{N}\right), \cdots, \theta_{N}\left(\theta_{1}, \alpha_{2}, \cdots, \alpha_{N}\right)\right) \mathbf{C}^{-1}
$$

system (1.9) can be rewritten in the equivalent form

$$
\mathbf{N}\left(\theta_{1}, \alpha_{2}, \cdots, \alpha_{N}\right)\left(\begin{array}{c}
\dot{\mathbf{x}}_{1} \\
\dot{\theta}_{1} \\
\dot{\alpha}_{2} \\
\vdots \\
\dot{\alpha}_{N}
\end{array}\right)=\left(\begin{array}{l}
0 \\
0 \\
0
\end{array}\right)
$$

We observe that we can decompose the $3 \times(N+2)$ matrix $\mathbf{N}\left(\theta_{1}, \alpha_{2}, \cdots, \alpha_{N}\right)$ in blocks into a $3 \times 3$ sub-matrix $\mathbf{A}\left(\theta_{1}, \alpha_{2}, \cdots, \alpha_{N}\right)$ and a $3 \times(N-1)$ sub-matrix $\mathbf{B}\left(\theta_{1}, \alpha_{2}, \cdots, \alpha_{N}\right)$, according to

$$
\mathbf{N}=(\mathbf{A} \mid \mathbf{B}) .
$$

The matrix A is known as the "grand-resistance-matrix" of a rigid system evolving at frozen shape, i.e., with $\dot{\alpha}_{i} \equiv 0, i=2, \ldots, N$, see [8]. it can be easily verified that it is symmetric and negative definite [8] and hence invertible. Therefore the equations of motion of the swimmer turn out to be affine system without drift. Indeed, solving (1.14) for $\left(\dot{\mathbf{x}}_{1}, \dot{\theta}_{1}\right)$ leads to 


$$
\left(\begin{array}{c}
\dot{\mathbf{x}}_{1} \\
\dot{\theta}_{1}
\end{array}\right)=-\mathbf{A}^{-1}\left(\theta_{1}, \alpha_{2}, \cdots, \alpha_{N}\right) \mathbf{B}\left(\theta_{1}, \alpha_{2}, \cdots, \alpha_{N}\right)\left(\begin{array}{c}
\dot{\alpha}_{2} \\
\vdots \\
\dot{\alpha}_{N}
\end{array}\right)
$$

which can be rewritten in the form

$$
\left(\begin{array}{c}
\dot{\mathbf{x}}_{1} \\
\dot{\theta}_{1}
\end{array}\right)=\sum_{i=2}^{N} \tilde{\mathbf{g}}_{i}\left(\theta_{1}, \alpha_{2}, \cdots, \alpha_{N}\right) \dot{\alpha}_{i}
$$

where the $N-1$ vector fields $\left\{\tilde{\mathbf{g}}_{i}\right\}_{i=2}^{N}$, are the columns of the $3 \times(N-1)$ matrix $-\mathbf{A}^{-1} \mathbf{B}$.

The equation above encode the link between the displacement (both translation and rotation) of the swimmer and its deformation. More precisely, prescribing the shape functions $t \mapsto\left(\alpha_{2}, \cdots, \alpha_{N}\right)(t)$, the motion of the swimmer is obtained by solving the system (1.16). In what follows we call stroke a time-periodic shape change, i.e., the functions $t \mapsto \alpha_{i}(t), i=2, \cdots, N$ are all periodic, with the same period.

In order to solve (1.16) numerically, we need to compute the vector fields $\tilde{\mathbf{g}}_{i}$ explicitly. To this end, we notice that the total force $\mathbf{F}$ and the total torque $\mathbf{T}_{\mathbf{x}_{1}}$ depend linearly on $\left(\dot{\mathbf{x}}_{i}\right)_{1 \leq i \leq N}$ and $\left(\dot{\theta}_{i}\right)_{1 \leq i \leq N}$ and that these quantities depend in turn linearly on $\left(\dot{\mathbf{x}}_{1}, \dot{\theta}_{1}, \cdots, \dot{\theta}_{N}\right)$ in view of (1.8). Therefore, we can rewrite (1.6) and (1.7) as

$$
\mathbf{F}=\mathbf{P}_{1}\left(\begin{array}{c}
\dot{\mathbf{x}}_{1} \\
\vdots \\
\dot{\mathbf{x}}_{N} \\
-- \\
\dot{\theta}_{1} \\
\vdots \\
\dot{\theta}_{N}
\end{array}\right)=\mathbf{P}_{1} \mathbf{Q}\left(\begin{array}{c}
\dot{\mathbf{x}}_{1} \\
\dot{\theta}_{1} \\
\vdots \\
\dot{\theta}_{N}
\end{array}\right), \mathbf{e}_{z} \cdot \mathbf{T}_{\mathbf{x}_{1}}=\mathbf{P}_{2}\left(\begin{array}{c}
\dot{\mathbf{x}}_{1} \\
\vdots \\
\dot{\mathbf{x}}_{N} \\
-- \\
\dot{\theta}_{1} \\
\vdots \\
\dot{\theta}_{N}
\end{array}\right)=\mathbf{P}_{2} \mathbf{Q}\left(\begin{array}{c}
\dot{\mathbf{x}}_{1} \\
\dot{\theta}_{1} \\
\vdots \\
\dot{\theta}_{N}
\end{array}\right),
$$

where

$$
\begin{gathered}
\mathbf{P}_{1}:=\left(-\mathbf{m}_{1} \cdots-\mathbf{m}_{N} \mid \frac{\eta}{2} L_{1}^{2} \mathbf{e}_{1}^{\perp} \cdots \frac{\eta}{2} L_{N}^{2} \mathbf{e}_{N}^{\perp}\right) \\
\text { with } \mathbf{m}_{i}:=L_{i}\left(\xi \mathbf{e}_{i} \otimes \mathbf{e}_{i}+\eta \mathbf{e}_{i}^{\perp} \otimes \mathbf{e}_{i}^{\perp}\right) \text { for } i=1 \cdots N \\
\mathbf{P}_{2}:=\left(\cdots-\left(L_{i}^{2} \eta \mathbf{e}_{i}^{\perp}+\left(\mathbf{x}_{i}-\mathbf{x}_{1}\right) \times \mathbf{m}_{i}\right)^{T} \cdots \mid \cdots \eta L_{i}^{2}\left(\frac{L_{i}}{3}+\frac{\left(\mathbf{x}_{i}-\mathbf{x}_{1}\right) \times \mathbf{e}_{i}^{\perp} \cdot \mathbf{e}_{z}}{2}\right) \cdots\right),
\end{gathered}
$$

and, finally, 


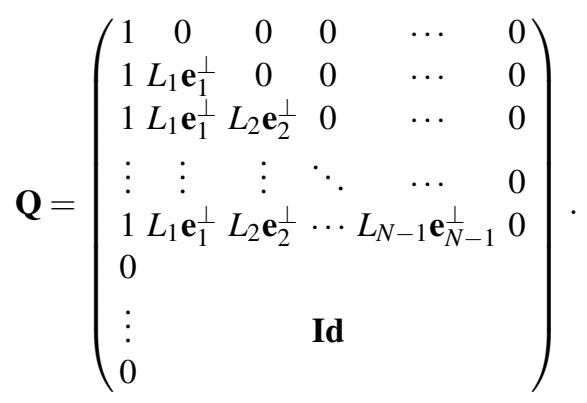

We thus have that the matrix $\mathbf{M}$ in (1.13) is

$$
\mathbf{M}=\left(\begin{array}{l}
\mathbf{P}_{1} \mathbf{Q} \\
\mathbf{P}_{2} \mathbf{q}
\end{array}\right)
$$

and we can compute $\mathbf{N}=\mathbf{C}^{-1} \mathbf{M}$, where $\mathbf{C}^{-1}$ is explicitly given as

$$
\mathbf{C}^{-1}=\left(\begin{array}{ccccccc}
1 & 0 & \cdots & \cdots & \cdots & \cdots & 0 \\
0 & 1 & \ddots & \ddots & \ddots & \ddots & \vdots \\
0 & 0 & 1 & \ddots & \ddots & & \vdots \\
0 & 0 & 1 & \ddots & \ddots & & \vdots \\
\vdots & \vdots & 1 & \ddots & \ddots & & 0 \\
\vdots & \vdots & \vdots & \ddots & \ddots & \ddots & 0 \\
0 & 0 & 1 & \cdots & 1 & 1 & 1
\end{array}\right)
$$

Matrices $\mathbf{A}$ and $\mathbf{B}$ are obtained from the columns of $\mathbf{N}$ as in (1.15) and, finally, the vectors $\tilde{\mathbf{g}}_{i}$ are simply the columns of $-\mathbf{A}^{-1} \mathbf{B}$. Finally the dynamics of the swimmer is expressed as

$$
\left(\begin{array}{c}
\dot{\alpha}_{2} \\
\vdots \\
\dot{\alpha}_{N} \\
\dot{\mathbf{x}}_{1} \\
\dot{\theta}_{1}
\end{array}\right)=\sum_{i=1}^{N-1}\left(\begin{array}{c}
\mathbf{b}_{i} \\
\tilde{\mathbf{g}}_{i}\left(\theta_{1}, \alpha_{2}, \cdots, \alpha_{N}\right)
\end{array}\right) \dot{\alpha}_{i+1}
$$

where $\mathbf{b}_{i}$ is the $i-$ th vector of the canonical basis of $\mathbf{R}^{N-1}$.

\subsection{Applications of the $N$-link swimmer}

The $N$-link swimmer model is very useful and can be used as a discrete approximation of a swimmer's flexible tail whose shape is controlled by curvature. We show in 
this section, how curvature control can be implemented in our model in a concrete case reproducing the motion of a sperm cell analyzed in [6].

\subsubsection{Curvature approximation}

Here, we describe the method to approximate the curvature of a beating tail with the discrete $N$ link swimmer model. Let $L>0$ be the total length of the flexible tail and let $\mathbf{r}(s, t)$ be the position, in the body frame of the swimmer (see Figure 1.2), at time $t>0$ of the point of arc-length coordinate $s \in[0, L]$ along the tail. We also define the angle between the tangent vector to the tail at the point $\mathbf{r}(s, t)$ and the $x$-axis in the lab-frame as $\Psi(s, t)$. It is wll known that the derivative of $\Psi(s, t)$ with respect to $s$ is the local curvature of the curve.

We discretize the swimmer's tail into $N$ equal parts of lenght $L_{i}=L / N$, and define the angles $\left(\theta_{i}\right)_{1 \leq i \leq N}$ by averaging the function $\Psi(s, t)$ on the interval $[i L / N,(i+1) L / N]$

$$
\theta_{i}(t)=\frac{N}{L} \int_{\frac{(i-1) L}{N}}^{\frac{i L}{L}} \Psi(s, t) d s, \quad i=1 \ldots N .
$$

Finally, differentiating (1.20) with respect to time we get the angular velocities $\dot{\theta}_{i}$, $i=1, \cdots, N$,

$$
\dot{\theta}_{i}(t)=\frac{N}{L} \int_{\frac{(i-1) L}{N}}^{\frac{i L}{N}} \frac{\partial \Psi(s, t)}{\partial t} d s, \quad i=1 \ldots N .
$$

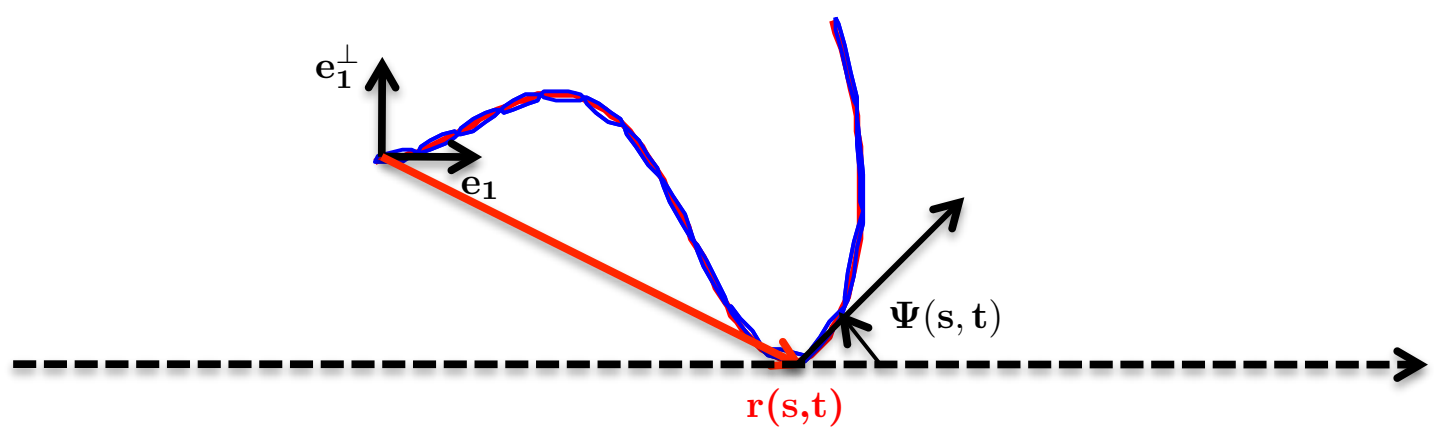

Fig. 1.2 The discrete approximation by the $N$-link swimmer (blue curve), $N=15$ of a continuous tail (red curve). 


\subsubsection{N-link approximation of sperm cell swimmer}

We now focus on reproducing with our model the motion of a sperm cell and compare to the one reported in [6]. To preform this comparison, we have to to take into account the presence of the head of the sperm cell. In order to do this, we modify the first segment of the $N$-link swimmer so that it has its own translational and rotational viscous drag. Indeed, we denote by $\mathbf{x}_{1}$ the position of the central point of the head and $\theta_{1}$ the angle that the direction of first segment (attached to the head) $\left(\mathbf{e}_{1}\right)$ makes with the horizontal axis. The movement of the head generates a viscous force and torque that are given by

$$
\mathbf{F}_{\text {head }}=-\xi_{\text {head }}\left(\dot{\mathbf{x}}_{1} \cdot \mathbf{e}_{1}\right) \mathbf{e}_{1}-\eta_{\text {head }}\left(\dot{\mathbf{x}}_{1} \cdot \mathbf{e}_{1}^{\perp}\right) \mathbf{e}_{1}^{\perp}
$$

and

$$
\mathbf{T}_{\text {head }} \cdot \mathbf{e}_{z}=-\zeta_{\text {head }} \dot{\theta}_{1} .
$$

We also fix that the length of the head is $L_{\text {head }}=10 \mu \mathrm{m}$ and we assume again that $L$ is the length of the tail which is attached to one of the extremities of the head segment. As suggested in [11], the wave profile along the tail of the sperm cell swimmer was obtained from experimental data, keeping only the two first Fourier modes, and we use the method described before in section 1.2.1 to approximate the tail's motion.

More precisely, we describe the shape of the wave shown in Fig. 1.3 by

$$
\mathbf{r}(s, t)=\frac{L_{\text {head }}}{2} \mathbf{e}_{1}(t)+\int_{0}^{s} \cos (\Psi(u, t)) \mathbf{e}_{1}(t)+\sin \left(\Psi(u, t) \mathbf{e}_{1}^{\perp}(t) d u .\right.
$$

where

$$
\Psi(s, t)=K_{0} s+2 A_{0} s \cos \left(\omega t-\frac{2 \pi s}{\lambda}\right) .
$$

In the previous equations, $K_{0}$ represents the mean flagellar curvature while $\omega, \lambda$ and $A_{0}$ are the frequency, the wave-length and the amplitude of the wave respectively. Following [6], in the next numerical simulations we use the following values for the wave parameters: $A_{0}=15.2 \cdot 10^{3} \mathrm{radm}^{-1}, K_{0}=19.1 \cdot 10^{3} \mathrm{radm}^{-1}, \omega=200 \mathrm{rad}, \mathrm{s}^{-1}$ and $\lambda=71.6 \cdot 10^{-6} \mathrm{~m}$.

Except the first segment, we discretize the rest of the tail with $N-1$ segments of extremities $\left(\mathbf{x}_{i}, \mathbf{x}_{i+1}\right)$ for $i=2, \cdots, N$. We use the method described in section 1.2.1, to approximate the beating wave and obtain the shapes shown in Figure 1.4 for one period $\left(0 \leq t \leq \frac{2 \pi}{\omega}\right)$. 


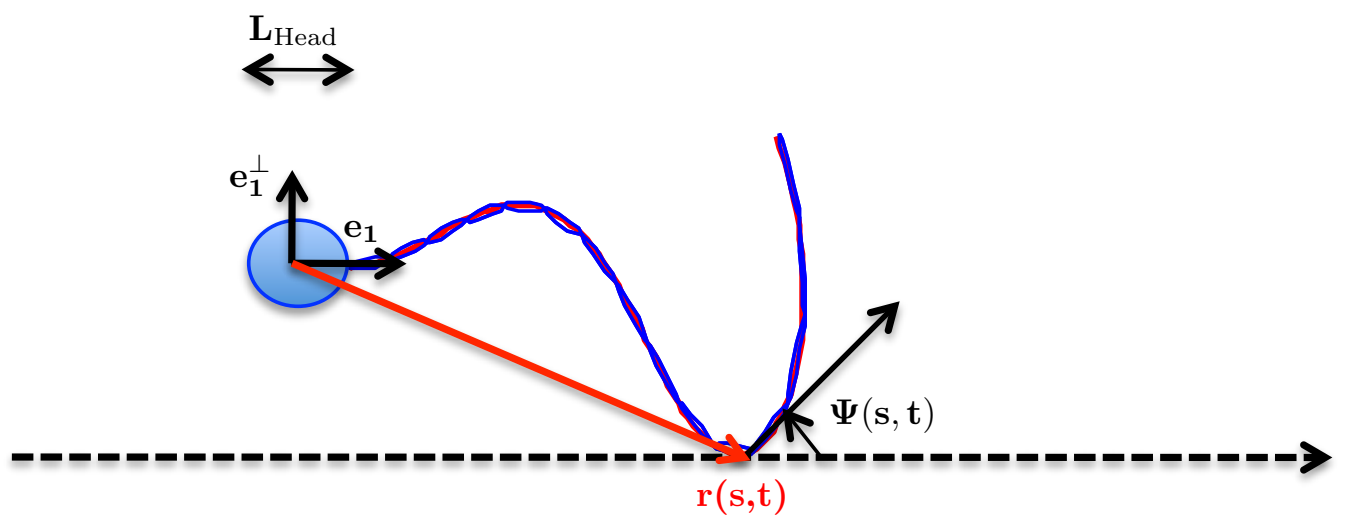

Fig. 1.3 The prescribed continuous wave (red curve) and its discrete approximation by the $N$-link swimmer (blue curve), $N=15$.

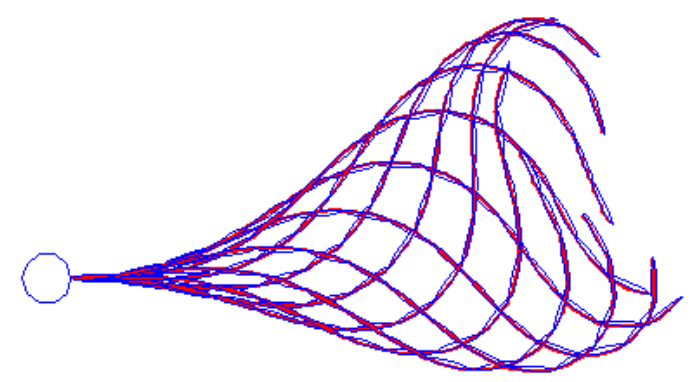

Fig. 1.4 Flagellar beating during one period. The red curve represents the tail as described by formula 1.24 while the blue links describe the tail according to our discrete approximation.

With the above notation, the equations of motion become

$$
\left\{\begin{array}{l}
\mathbf{F}=\mathbf{F}_{\text {head }}+\sum_{i=1}^{N} \int_{0}^{L_{i}} \mathbf{f}_{i}(s) d s, \\
\mathbf{T}_{\mathbf{x}_{1}}=\mathbf{T}_{\text {head }}+\sum_{i=1}^{N} \int_{0}^{L_{i}} \mathbf{f}_{i}(s) \times\left(\mathbf{x}_{i}(s)-\mathbf{x}_{1}\right) d s .
\end{array}\right.
$$

where $L_{i}=L / N$ is the length of each segment $\left(\mathbf{x}_{i}, \mathbf{x}_{i+1}\right)$ for $i=2, \cdots, N$, while the first segment, also of size $L_{1}=L / N$ is given by $\left(\mathbf{x}_{1}+\frac{L_{\text {head }}}{2} \mathbf{e}_{1}, \mathbf{x}_{2}\right)$.

Since the two previous formulas (1.26) are linear in $\dot{\theta}_{1}$ and $\dot{\mathbf{x}}_{1}$, we end up with the same compact expression of the equations of motion (1.16). More precisely, the matrix $\mathbf{P}_{1}$ and $\mathbf{P}_{2}$ defined in system (1.17) are replaced by 


$$
\mathbf{P}_{1}^{\text {head }}:=\left(-\xi_{\text {head }} \mathbf{e}_{1} \otimes \mathbf{e}_{1}+\eta_{\text {head }} \mathbf{e}_{1}^{\perp} \otimes \mathbf{e}_{1}^{\perp}-\mathbf{m}_{1} \cdots-\mathbf{m}_{N} \mid \frac{\eta}{2} L_{1}^{2} \mathbf{e}_{1}^{\perp} \cdots \frac{\eta}{2} L_{N}^{2} \mathbf{e}_{N}^{\perp}\right)
$$

and

$$
\mathbf{P}_{2}^{\text {head }}:=\left(-p_{1} \cdots-p_{N} \mid-\zeta_{\text {head }}+q_{1} q_{2} \cdots q_{N}\right),
$$

with $\mathbf{m}_{i}:=L_{i}\left(\xi \mathbf{e}_{i} \otimes \mathbf{e}_{i}+\eta \mathbf{e}_{i}^{\perp} \otimes \mathbf{e}_{i}^{\perp}\right)$ for $i=1 \cdots N$, and $p_{i}:=\left(L_{i}^{2} \eta \mathbf{e}_{i}^{\perp}+\left(\mathbf{x}_{i}-\mathbf{x}_{1}\right) \times\right.$ $\left.\mathbf{m}_{i}\right)^{T}, q_{i}:=\eta L_{i}^{2}\left(\frac{L_{i}}{3}+\frac{\left(\mathbf{x}_{i}-\mathbf{x}_{1}\right) \times \mathbf{e}_{i}^{\perp} \cdot \mathbf{e}_{z}}{2}\right)$, for $i=1 \cdots N$.

Moreover we use the following values for the drag coefficients

- for the head, $\xi_{\text {head }}=40.3 \cdot 10^{3} \mathrm{pNsm^{-1 }}, \eta_{\text {head }}=46.1 \cdot 10^{3} \mathrm{pNsm} \mathrm{sm}^{-1}$, and $\zeta_{\text {head }}=$ $0.84 \cdot 10^{-6} \mathrm{pNsm}$

- for the links rcomposing the tail, $\xi=0.38 \cdot 10^{9} \mathrm{p} N \mathrm{sm}^{-2}, \frac{\eta}{\xi}=1.89$.

The graphs in Figures 1.5 and 1.6 below, summarize our results that are in perfect agreement with those of [6] (see Figure 3 for the trajectory and Figure 4 for the various speeds).
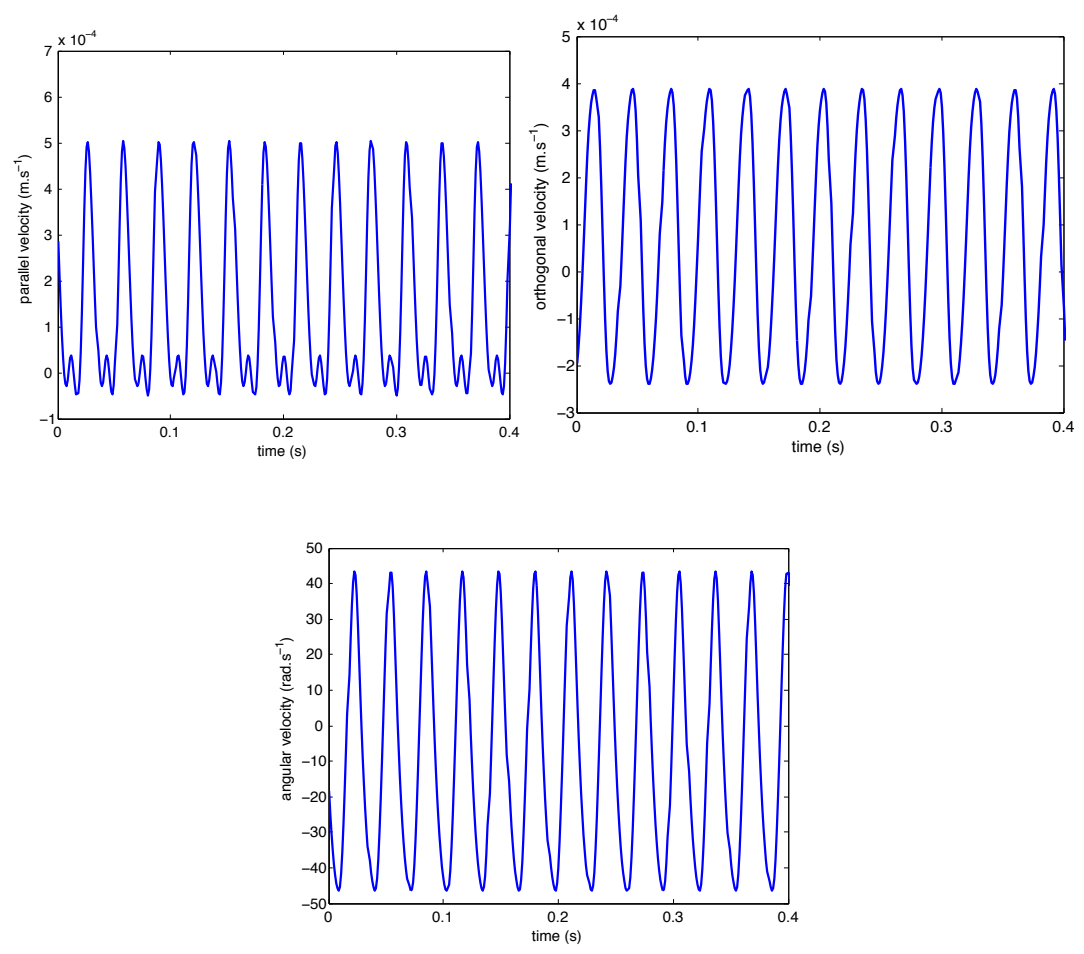

Fig. 1.5 Above translational speed of the swimmer head in the tangent and perpendicular directions, and below rotational speed. 


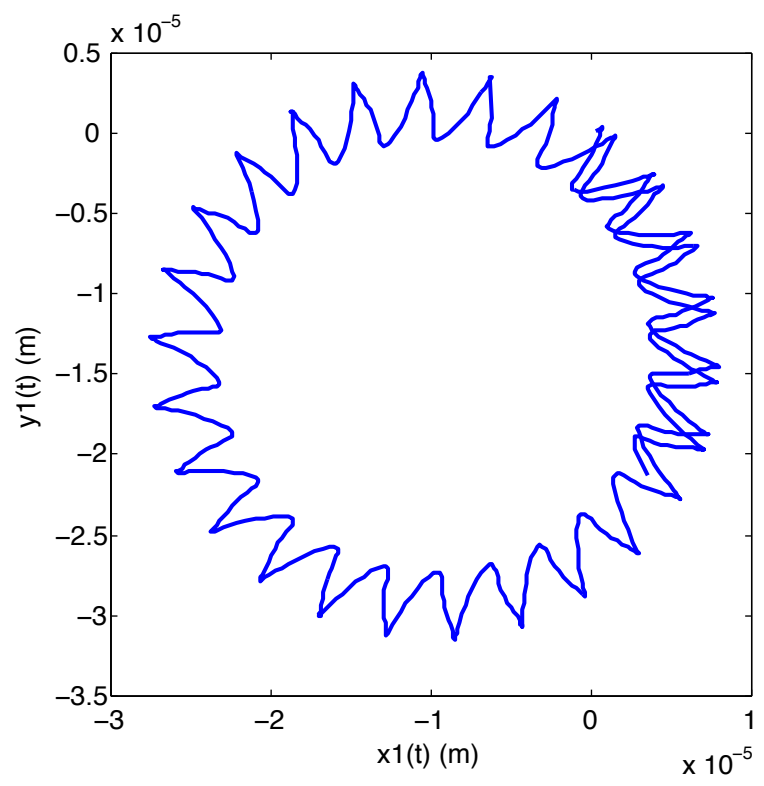

Fig. 1.6 Trajectory of the head of the sperm-cell during one period.

\subsection{Controllability}

This section is devoted to the controllability of the $N$-link swimmer, which is its ability to move between two fixed configurations prescribing (controlling) its shape parameters. More precisely we prove that there exist control shape functions which allow the swimmer to move everywhere in the plane.

Theorem 1.1. Consider the N-link swimmer described in section 1.1 evolving in the space $\mathbf{R}^{2}$. Then for almost every lengths of the sticks $\left(L_{i}\right)_{i=1, \cdots, N}$ and for any initial configuration $\left(\mathbf{x}_{1}^{i}, \theta_{1}^{i}, \alpha_{2}^{i}, \cdots, \alpha_{N}^{i}\right) \in \mathbf{R}^{2} \times[0,2 \pi]^{N}$, any final configuration $\left(\mathbf{x}_{1}^{f}, \theta_{1}^{f}, \alpha_{2}^{f}, \cdots, \alpha_{N}^{f}\right)$ and any final time $T>0$, there exists a shape function $\left(\alpha_{2}, \cdots, \alpha_{N}\right) \in \mathscr{W}^{1, \infty}([0, T])$, satisfying $\left(\alpha_{2}, \cdots, \alpha_{N}\right)(0)=\left(\alpha_{2}^{i}, \cdots, \alpha_{N}^{i}\right)$ and $\left(\alpha_{2}, \cdots, \alpha_{N}\right)(T)=\left(\alpha_{2}^{f}, \cdots, \alpha_{N}^{f}\right)$ and such that if the self-propelled swimmer starts in position $\left(\mathbf{x}_{1}^{i}, \theta_{1}^{i}\right)$ with the shape $\left(\alpha_{2}^{i}, \cdots, \alpha_{N}^{i}\right)$ at time $t=0$, it ends at position $\left(\mathbf{x}_{1}^{f}, \theta_{1}^{f}\right)$ and shape $\left(\alpha_{2}^{f}, \cdots, \alpha_{N}^{f}\right)$ at time $t=T$ by changing its shape along $\left(\alpha_{2}, \cdots, \alpha_{N}\right)(t)$.

Proof. The proof of the theorem is divided into three steps. First of all, we show the analyticity of the dynamics vector fields. Then, we prove the controllability of 
the 3-link swimmer (Purcell swimmer), exploiting the Chow theorem and the Orbit theorem. Finally, we generalize the result to the case of $N$ links. We start by recalling some classical results used in the proof.

\subsubsection{Classical results in geometric control}

Theorem 1.2. (Chow (see [5])) Let $m, n \in \mathbb{N}$ and let $\left(\mathbf{g}_{i}\right)_{i=1, n}$ be $\mathscr{C}^{\infty}$ vector fields on $\mathbb{R}^{n}$. Consider the control system, of state trajectory $\mathbf{q}$,

$$
\dot{\mathbf{q}}=\sum_{i=1}^{m} u_{i} \mathbf{g}_{i}(\mathbf{q})
$$

with input function $\mathbf{u}=\left(u_{i}\right)_{i=1, m} \in L^{\infty}\left(\left[0,+\infty\left[, \mathbf{B}_{\mathbb{R}^{n}}(0, \delta)\right)\right.\right.$ for some $\boldsymbol{\delta}>0$.

Let $\mathscr{O}$ an open and connected set of $\mathbb{R}^{n}$ and assume that

$$
\mathbf{L i e}_{\mathbf{q}}\left(\mathbf{g}_{1}, \ldots \mathbf{g}_{m}\right)=\mathbb{R}^{n} \mathbf{q} \in \mathscr{O}
$$

Then the system (1.27) is controllable, i.e., for every $\mathbf{q}_{0}, \mathbf{q}_{1}$ in $\mathscr{O}$ and for every $T>0$ exists $\mathbf{u} \in L^{\infty}\left((0, T), \mathbf{B}_{\mathbb{R}^{n}}(0, \delta)\right)$ such that $\mathbf{q}(0)=\mathbf{q}_{0}$ and $\mathbf{q}(T)=\mathbf{q}_{1}$ and $\mathbf{q}(t) \in \mathscr{O}$ for every $t \in[0, T]$.

If the vector fields are analytic, we can apply the Orbit Theorem to extend the dimension property of the Lie algebra defined by the dynamics vector fields on the whole orbit.

Theorem 1.3. (Orbit (see [9]) Let $\mathscr{M}$ be an analytic manifold, and $\mathscr{F}$ a family of analytic vector fields on $\mathscr{M}$. Then

a) each orbit of $\mathscr{F}$ is an analytic submanifold of $\mathscr{M}$, and

b) if $N$ is an orbit of $\mathscr{F}$, then the tangent space of $N$ at $x$ is given by $\operatorname{Lie}_{x}(\mathscr{F})$. In particular the dimension of $\operatorname{Lie}_{x}(\mathscr{F})$ is constant as $x$ varies on $N$.

In our case, the manifold in which the state and the shape of the swimmer evolve is defined by $\mathscr{M}:=[0,2 \pi]^{N-1} \times \mathbb{R}^{2} \times[0,2 \pi]$. The vector fields of the dynamics are denoted by

$$
\mathbf{g}_{i}\left(\theta_{1}, \alpha_{2}, \cdots, \alpha_{N}\right):=\left(\begin{array}{c}
\mathbf{b}_{i} \\
\tilde{\mathbf{g}_{i}}\left(\theta_{1}, \alpha_{2}, \cdots, \alpha_{N}\right)
\end{array}\right) .
$$

We say that the Lie algebra of the family of vector fields $\left\{\mathbf{g}_{i}\right\}_{i=1, \cdots, N-1}$ is fully generated at the point $\mathbf{q}=\left(\alpha_{2}, \cdots, \alpha_{N}, x_{1}, y_{1}, \theta_{1}\right) \in \mathscr{M}$ if the tangent space of the manifold, $T_{\mathbf{q}} \mathscr{M}$, is equal to the Lie algebra $\left.\operatorname{Lie}\left(\left(\mathbf{g}_{i}\right)\right)_{i=1, \cdots, N-1}\right)(\mathbf{q})$. 


\subsubsection{Regularity}

We first prove that the vector fields $\left(\tilde{\mathbf{g}}_{i}\right)$ are analytic on $\mathscr{M}$. From (1.6) and (1.7), the entries of the matrices $\mathbf{A}$ and $\mathbf{B}$ are analytic functions on $[0,2 \pi]^{N}$. Since the coefficients of $\mathbf{A}^{-1}$ are obtained by multiplication and division of those of $\mathbf{A}$, and because $\operatorname{det}(\mathbf{A}) \neq 0$ ( $\mathbf{A}$ is symmetric and negative defined), the entries of $\mathbf{A}^{-1}$ remain analytic functions on $[0,2 \pi]^{N}$. Thus, the $\left(\tilde{\mathbf{g}}_{i}\right)_{i=1, \cdots, N}:=\mathbf{A}^{-1} \mathbf{B}$ are analytic on $[0,2 \pi]^{N}$.

\subsubsection{Controllability of the Purcell Swimmer (N=3)}

Setting $N=3$ in (1.19) the dynamics becomes

$$
\left(\begin{array}{c}
\dot{\alpha}_{2} \\
\dot{\alpha}_{3} \\
\dot{x}_{1} \\
\dot{y}_{1} \\
\dot{\theta}_{1}
\end{array}\right)=\mathbf{g}_{1}\left(\theta_{1}, \alpha_{2}, \alpha_{3}\right) \dot{\alpha}_{2}+\mathbf{g}_{2}\left(\theta_{1}, \alpha_{2}, \alpha_{3}\right) \dot{\alpha}_{3}
$$

To prove the controllability of this system we want use Theorem 1.2.

Therefore we compute the Lie algebra of the vector fields $\mathbf{g}_{1}$ and $\mathbf{g}_{2}$ for any $\theta_{1} \in$ $[0,2 \pi]$ at $\left(\alpha_{2}, \alpha_{3}\right)=(0,0)$, for a swimmer whose sticks have the length $L_{1}=L_{3}=L$ and $L_{2}=2 L$ where $L>0$. First we have

$$
\mathbf{g}_{1}\left(\theta_{1}, 0,0\right)=\left(\begin{array}{c}
1 \\
0 \\
\frac{9 L \sin \left(\theta_{1}\right)}{64} \\
-\frac{9 L \cos \left(\theta_{1}\right)}{64} \\
\frac{27}{32}
\end{array}\right) \mathbf{g}_{2}\left(\theta_{1}, 0,0\right)=\left(\begin{array}{c}
0 \\
1 \\
-\frac{7 L \sin \left(\theta_{1}\right)}{64} \\
\frac{7 L \cos \left(\theta_{1}\right)}{64} \\
-\frac{5}{32}
\end{array}\right)
$$

Then, the iterated Lie brackets are equals to

$$
\begin{gathered}
{\left[\mathbf{g}_{1}, \mathbf{g}_{2}\right]\left(\theta_{1}, 0,0\right)=\left(0,0, \frac{7 L(\eta-\xi) \cos \left(\theta_{1}\right)}{128 \xi}, \frac{7 L(\eta-\xi) \sin \left(\theta_{1}\right)}{128 \xi}, 0\right)^{T},} \\
0 \\
0 \\
{\left[\mathbf{g}_{1},\left[\mathbf{g}_{1}, \mathbf{g}_{2}\right]\right]\left(\theta_{1}, 0,0\right)=\left(\begin{array}{c}
-\frac{L\left(126 \eta^{2}+31 \xi \eta-76 \xi^{2}\right) \sin \left(\theta_{1}\right)}{4096 \eta \xi} \\
\frac{L\left(126 \eta^{2}+31 \xi \eta-76 \xi^{2}\right) \cos \left(\theta_{1}\right)}{4096 \eta \xi} \\
-\frac{3\left(9 \eta^{2}-4 \xi \eta+4 \xi^{2}\right)}{2048 \eta \xi}
\end{array}\right),}
\end{gathered}
$$




$$
\left[\mathbf{g}_{2},\left[\mathbf{g}_{1}, \mathbf{g}_{2}\right]\right]\left(\theta_{1}, 0,0\right)=\left(\begin{array}{c}
0 \\
0 \\
\frac{L\left(36 \eta^{2}-103 \xi \eta+148 \xi^{2}\right) \sin \left(\theta_{1}\right)}{4096 \eta \xi} \\
-\frac{L\left(36 \eta^{2}-103 \xi \eta+148 \xi^{2}\right) \cos \left(\theta_{1}\right)}{4096 \eta \xi} \\
\frac{3\left(9 \eta^{2}-4 \xi \eta+4 \xi^{2}\right)}{2048 \eta \xi}
\end{array}\right) .
$$

To see if they are linearly independent we compute the determinant of the matrix whose columns are the 5 previous vector fields that is equal to

$$
\frac{21 L^{2}(\eta-\xi)^{2}(45 \eta+112 \xi)\left(9 \eta^{2}-4 \eta \xi+4 \xi^{2}\right)}{536870912 \eta^{2} \xi^{3}} .
$$

Since the drag coefficients $\xi$ and $\eta$ are positive, this determinant is null only when $\xi=\eta$. This would imply an isotropic drag, as we would have if we use spheres instead of sticks. Thus in our case the Lie algebra of the vector fields $\mathbf{g}_{1}$ and $\mathbf{g}_{2}$ is fully generated at the point $\left(\theta_{1}, 0,0\right)$, for any $\theta_{1} \in[0,2 \pi]$.

Notice that any point $\left(\alpha_{2}, \alpha_{3}, \mathbf{x}_{1}, \theta_{1}\right) \in[0,2 \pi]^{2} \times \mathbf{R}^{2} \times[0,2 \pi]$ belongs to the orbit of the point $\left(0,0, \mathbf{x}_{1}, \theta_{1}\right)$. Since the vector fields are analytic, the Orbit Theorem 1.3 guarantees that the Lie algebra of $\mathbf{g}_{1}$ and $\mathbf{g}_{2}$ is fully generated everywhere in the manifold $\mathscr{M}=[0,2 \pi]^{2} \times \mathbf{R}^{2} \times[0,2 \pi]$.

To conclude, by Chow Theorem (1.2) we get the controllability of the Purcell swimmer.

\subsubsection{Controllability of the N-link swimmer}

The third step is to generalize the previous controllability result to the $N$-link swimmer, whose dynamics is described by (1.19). It is easy to see that the vector fields $\mathbf{g}_{i}$ generate the tangent space of the manifolds $[0,2 \pi]^{N-1}$,

$$
\operatorname{Span}\left(\mathbf{g}_{1}, \cdots, \mathbf{g}_{N-1}\right)=\mathbf{R}^{N-1} \text {. }
$$

We can obtain the vector fields $\mathbf{g}_{1}$ and $\mathbf{g}_{2}$ starting from the Purcell's one defined in (1.28) as follows: we add $N-2$ rows of zeroes, take sticks of null length $L_{i}=0$ for $4 \leq i \leq N-1$, while keeping the three sticks $L_{1}=L_{3}=L$ and $L_{2}=2 L$.

In this case, for any $\left(\mathbf{x}_{1}, \theta_{1}\right) \in \mathbf{R}^{2} \times[0,2 \pi]$, subsection 1.3.3 shows that $\mathbf{g}_{1}\left(\theta_{1}, 0, \cdots 0\right)$, $\mathbf{g}_{2}\left(\theta_{1}, 0, \cdots 0\right)$ and their iterated Lie brackets $\left[\mathbf{g}_{1}, \mathbf{g}_{2}\right]\left(\theta_{1}, 0, \cdots 0\right),\left[\mathbf{g}_{1},\left[\mathbf{g}_{1}, \mathbf{g}_{2}\right]\right]\left(\theta_{1}, 0, \cdots 0\right)$, and $\left[\mathbf{g}_{2},\left[\mathbf{g}_{1}, \mathbf{g}_{2}\right]\right]\left(\theta_{1}, 0, \cdots 0\right)$ are linearly independent.

Therefore, the Lie algebra of the family $\left(\mathbf{g}_{i}\right)_{i=1, \cdots, N-1}$ at the point $\left(\theta_{1}, 0, \cdots, 0\right)$ is 
equal to the tangent space $T_{\left(0, \cdots, 0, \mathbf{x}_{1}, \theta_{1}\right)} \mathscr{M}$. Then, by analyticity of the vector fields $\mathbf{g}_{i}$, the Orbit Theorem ensures that the Lie algebra is fully generated everywhere for a swimmer whose length of sticks verify $L_{1}=L_{3}=L, L_{2}=2 L$ and $L_{i \geq 4}=0$.

We call $D^{(0, \cdots, 0)}$, the function that maps $\left(L_{1}, \cdots, L_{N}\right)$ to the determinant of the vectors $\mathbf{g}_{1}, \cdots, \mathbf{g}_{N-1}$ and their iterated Lie brakets at the point $(0, \cdots, 0)$. Since the vector fields $\mathbf{g}_{i}$ depend analytically on the sticks length $L_{i}$, we get the analyticity of the function $D^{(0, \cdots, 0)}$. Thus for any $L>0$, the value of $D^{(0, \cdots, 0)}$ at the point $(L, 2 L, L, 0 \cdots 0)$ is not null. By analyticity, it remains non null almost everywhere in $\mathbf{R}^{N}$. Therefore, we obtain that the Lie algebra has full rank for almost every swimmer.

Finally, Chow Theorem gives the controllability in the Theorem 1.1.

\subsection{Minimum time optimal control problem for the $N$-link swimmer}

We present in subsection 1.4.1 the minimum time optimal control problem for the $N$-link swimmer, which is well defined from the controllability result proven in section 1.3. Then in subsection 1.4.2 we present the numerical method used to solve this problem.

\subsubsection{Minimum Time Problem}

For any time $t>0$, we use the following notation: the state of the swimmer is $\mathbf{z}(t):=\left(\alpha_{2}, \cdots, \alpha_{N}, \mathbf{x}_{1}, \theta_{1}\right)(t)$, the control functions are $\mathbf{u}(t):=\left(\dot{\alpha}_{2}, \cdots, \dot{\alpha}_{N}\right)(t)$ and the dynamics is $\mathbf{f}(\mathbf{z}(t), \mathbf{u}(t))=\sum_{i=1}^{N-1} \mathbf{g}_{i}(\mathbf{z}(t)) \dot{\alpha}_{i+1}(t)$.

We now assume that the swimmer starts at the initial configuration $\mathbf{z}^{i}$, and we fix a final state $\mathbf{z}^{f}$. Our aim is to find a swimming strategy that minimizes the time to swim between the initial and the final configuration, i.e.,

$$
(O C P)\left\{\begin{array}{l}
\inf t_{f}, \\
\dot{\mathbf{z}}(t)=\mathbf{f}(\mathbf{z}(t), \mathbf{u}(t)), \forall t \in\left[0, t_{f}\right] \\
\mathbf{u}(t) \in \mathbf{U}:=[-1,1]^{N}, \forall t \in\left[0, t_{f}\right], \\
\mathbf{z}(0)=\mathbf{z}^{i}, \quad \mathbf{z}\left(t_{f}\right)=\mathbf{z}^{f}
\end{array}\right.
$$

By applying Filippov-Cesary Theorem ([12]) which ensures the existence of a solution of the minimum time problem for controllable systems, there exists a minimal time such that the constraints are satisfied i.e., the infimum can be written as a minimum. 


\subsubsection{Numerical Optimization}

In order to solve this optimal control problem, we use a direct approach. This approach transforms the infinite dimensional optimal control problem $(O C P)$ into a finite dimensional optimization problem $(N L P)$. This is done with a discretization procedure on the dynamics equation summarized below:

$$
\begin{aligned}
& t \in[0, t f] \rightarrow\left\{t_{0}=0, \ldots, t_{N}=t f\right\} \\
& z(\cdot), u(\cdot) \rightarrow X=\left\{z_{0}, \ldots, z_{N}, u_{0}, \ldots, u_{N-1}, t f\right\} \\
& \hline \text { Criterion } \rightarrow \min t f \\
& \text { Dynamics } \rightarrow(\text { ex }: \text { Euler }) z_{i+i}=z_{i}+h f\left(z_{i}, u_{i}\right) \\
& \text { Controls } \rightarrow-1 \leq u_{i} \leq 1 \\
& I / F \text { Cond. } \rightarrow \Phi\left(z_{0}, z_{N}\right)=0
\end{aligned}
$$

We therefore obtain a nonlinear programming problem on the discretized state and control variables

$$
(N L P)\left\{\begin{array}{l}
\min F(z)=t f \\
L B \leq C(z) \leq U B
\end{array}\right.
$$

All tests were run using the software BocOP ([4]). The discretized nonlinear optimization problem is solved by the well-known solver IPOPT [13] with MUMPS [2], while the derivatives are computed by sparse automatic differentiation with ADOL$\mathrm{C}$ [14] and COLPACK [7]. In the numerical experiments, we used a Midpoint (implicit 2nd order) discretization with 1000 time steps. Execution times on a Xeon 3.2GHz CPU were a few minutes.

\subsection{Numerical simulations for the Purcell's swimmer $(\mathbf{N}=3)$}

We present in this section the numerical simulations regarding the Purcell swimmer (3 links). Without making any assumptions on the structure of the optimal trajectory, we obtain an optimal solution with periodic strokes. Comparing this stroke to the one of Purcell ([10], [3]), we observe that it gives a better displacement speed.

In the rest of the chapter, in order to match the notations used in [3], we we the following coordinates (see Fig 1.7):

- the position $\left(x_{2}, y_{2}\right)$ of the center of the second stick, and its angle with the $\mathrm{x}$-axis $\theta_{2}:=\theta_{1}-\alpha_{2}$.

- the shape angles $\beta_{1}:=-\alpha_{2}$ and $\beta_{3}:=\alpha_{3}$. 


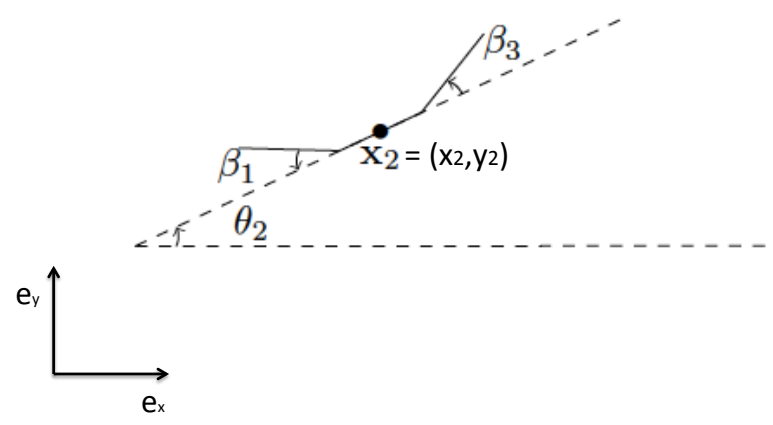

Fig. 1.7 Purcell's 3-link swimmer.

This reformulation gives the new dynamics

$$
\begin{aligned}
& \left(\begin{array}{c}
\dot{\beta}_{1} \\
\dot{\beta}_{3} \\
\dot{\mathbf{x}}_{2} \\
\dot{\theta}_{2}
\end{array}\right)=\mathbf{M}\left(\theta_{2}, \beta_{1}\right)\left(\begin{array}{c}
\dot{\alpha}_{2} \\
\dot{\alpha}_{3} \\
\dot{\mathbf{x}}_{1} \\
\dot{\theta}_{1}
\end{array}\right), \\
\mathbf{M}\left(\theta_{2}, \beta_{1}\right)= & \left(\begin{array}{ccccc}
-1 & 0 & 0 & 0 & 0 \\
0 & 1 & 0 & 0 & 0 \\
\sin \left(\theta_{2}\right)+\cos \left(\beta_{1}\right) & 0 & 1 & 0 & -\sin \left(\theta_{2}\right) \\
-\cos \left(\beta_{1}\right)-\cos \left(\theta_{2}\right) & 0 & 0 & 1 & \cos \left(\theta_{2}\right) \\
-1 & 0 & 0 & 0 & 1
\end{array}\right) .
\end{aligned}
$$

As a result, the dynamics (1.19) reads in this case as

$$
\left(\begin{array}{c}
\dot{\beta}_{1} \\
\dot{\beta}_{3} \\
\dot{\mathbf{x}}_{2} \\
\dot{\theta}_{2}
\end{array}\right)=\tilde{\mathbf{f}}_{1}\left(\theta_{2}, \beta_{2}, \beta_{3}\right) \dot{\beta}_{1}+\tilde{\tilde{f}_{2}}\left(\theta_{2}, \beta_{2}, \beta_{3}\right) \dot{\beta}_{3}
$$

where for $i=1,2$

$$
\tilde{\mathbf{f}}_{i}\left(\theta_{2}, \beta_{1}, \beta_{3}\right)=\mathbf{M}\left(\theta_{2}, \beta_{1},\right) \tilde{\mathbf{g}}_{i}\left(\theta_{1}, \alpha_{2}, \alpha_{3}\right)
$$

Observe that since the new state variables are the image of the former ones through a one-to-one mapping, the controllability result in subsection 1.3.3 holds also for (1.31). 


\subsubsection{The classical Purcell stroke}

We recall the stroke presented by Purcell in [10] in order to compare it to the optimal strategy given by our numerical results. Let us denote by $\Delta \theta$ the angular excursion, which means that $\beta_{1}$ and $\beta_{3}$ belong to $\left[-\frac{\Delta \theta}{2}, \frac{\Delta \theta}{2}\right]$. The Purcell stroke is defined by this periodic of deformation over $[0, T]$ :

$$
\left(\beta_{1}(t), \beta_{3}(t)\right)=\left\{\begin{array}{cc}
\left(\frac{4 \Delta \theta}{T} t-\frac{\Delta \theta}{2}, \frac{\Delta \theta}{2}\right) & 0 \leq t \leq \frac{T}{4} \\
\left(\frac{\Delta \theta}{2},-\frac{4 \Delta \theta}{T} t+\frac{3 \Delta \theta}{2}\right) & \frac{T}{4} \leq t \leq \frac{T}{2} \\
\left(-\frac{4 \Delta \theta}{T} t+\frac{5 \Delta \theta}{2},-\frac{\Delta \theta}{2}\right) & \frac{T}{2} \leq t \leq \frac{3 T}{4} \\
\left(-\frac{\Delta \theta}{2}, \frac{4 \Delta \theta}{T} t-\frac{7 \Delta \theta}{2}\right) & \frac{3 T}{4} \leq t \leq T
\end{array}\right.
$$

In what follows, we call the "classical" Purcell stroke the one corresponding to $\Delta \theta=\frac{\pi}{3}$, with $T=4 \Delta \theta$ chosen in order to satisfy the constraints on the controls of (OCP), i.e., $u_{i}(t):=\dot{\beta}_{i}(t) \in[-1,1]$.

\subsubsection{Comparison of the optimal stroke and Purcell stroke}

We set the initial position $\left(\mathbf{x}_{2}, \theta_{2}\right)=(0,0,0)$ and the final position $\left(\mathbf{x}_{2}, \theta_{2}\right)=$ $(-0.25,0,0)$. Moreover we constrain the angles $\beta_{1}$ and $\beta_{3}$ in $\left[-\frac{\pi}{6}, \frac{\pi}{6}\right]$ for all time. Solving the minimum time problem numerically with the direct method gives us a solution that is actually periodic, as shown on Fig. 1.8. We notice that the $\mathrm{x}$ displacement is not monotone: during each stroke, the swimmer alternately moves forward, closer to the target, and goes partially backward.
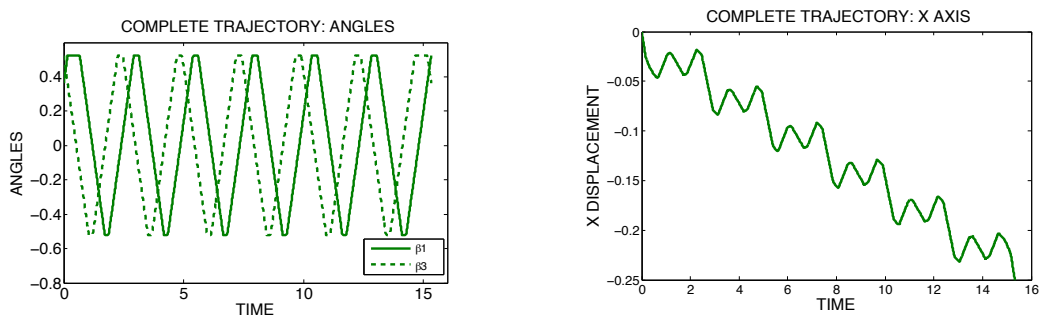

Fig. 1.8 Angles and $x$-displacement for a whole periodic trajectory.

Now we isolate only one stroke from this solution, and compare it with the Purcell stroke. We show on Fig. 1.9 the angles functions $\beta_{1}$ and $\beta_{3}$, as well as the phase portrait. Note that the time required to complete our candidate for an optimal stroke is shorter than for the Purcell one (roughly 2.5 versus 4.1). We illustrate on Fig. 1.10 the shape changes in the plane for the Purcell and optimal stroke. 

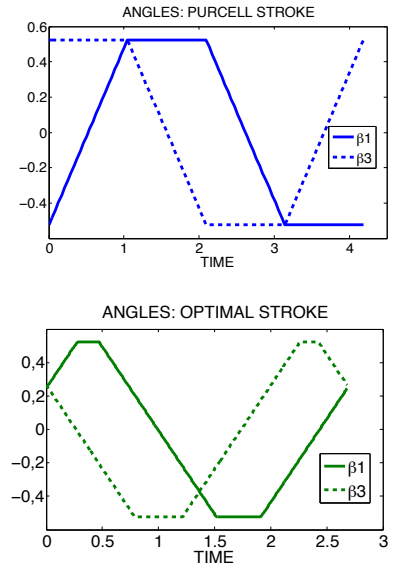

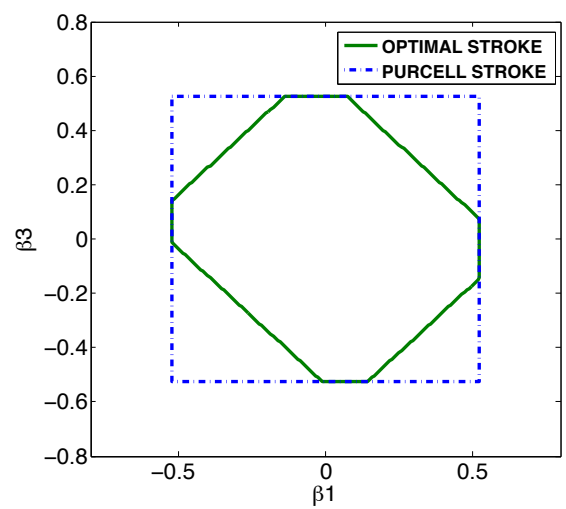

Fig. 1.9 Angles and phase portrait - Purcell stroke and optimal stroke.
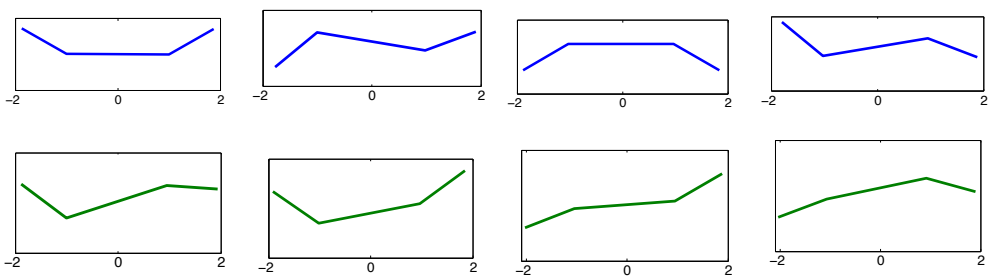

Fig. 1.10 Purcell stroke (above) and optimal stroke (below).

Finally we make a comparison between the two $x$-displacement, Fig. 1.11 shows the $x$-displacement of the swimmer with the classical Purcell stroke (dashed) and the optimal stroke (solid). Both trajectories were recomputed in Matlab using the same ODE solver, and the results for the Purcell stroke match the ones in [3]. The final time $t_{f}=15.3252$ is the one given by the numeric simulation to reach $\mathbf{x}_{2}=(-0.25,0)$. We see that using Purcell strokes, the swimmer only reaches $(\approx-0.18,0)$, which confirms that our optimal stroke allows a greate $x$ displacement.

More precisely, each optimal stroke gives a $x$-displacement close to the Purcell stroke, however the cycle of deformation is performed in less time. Therefore, for a given time frame, more optimal strokes can be performed, leading to an overall greater displacement. In Fig. 1.11, almost 3.5 Purcell strokes are performed, while 6 optimal strokes are completed within the same time. 


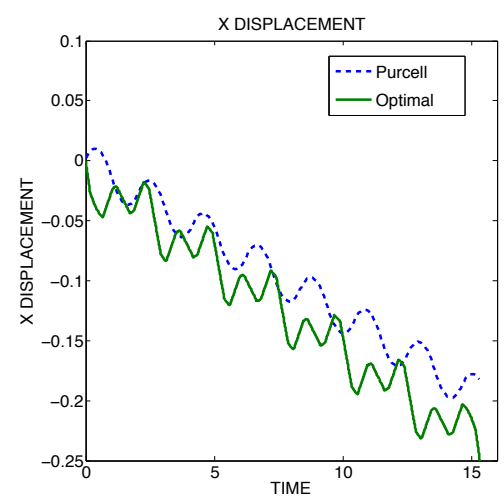

Fig. 1.11 x displacement for one Purcell and one optimal stroke.

Remark: The initial shape of the swimmer is not identical for both strategies, however the increasing gap between the two curves clearly shows that the optimal stroke is faster.

We also observe that the optimal stroke consistently gives a swimming speed better by $20 \%$ than the Purcell stroke.

\subsection{Conclusions}

In this chapter we study the $N$-link swimmer, and use the Resistive Force Theory to derive its dynamics. In this context, we prove that for $N$ greater than 3 and for almost any $N$-uplet of sticks lengths, the swimmer is globally controllable in the whole plane. Then, we focus on finding a swimming strategy that leads the $N$-link swimmer from an fixed initial position to a given final position, in minimum time. As a consequence of the controllability result, we show that there exists a shape change function which allows to reach the final state in a minimal time. We formulate this optimal control problem and solve it with a direct approach (BOCOP) for the case $N=3$ (Purcell swimmer). Without any assumption on the structure of the trajectory, we obtain a periodic solution, from which we identify an optimal stroke. Comparing this optimal stroke with the Purcell one confirms that it is better, actually giving a greater displacement speed. 


\section{References}

1. Alouges, F., DeSimone, A., Heltai, L., Lefebvre, A., Merlet, B.: Optimally swimming stokesian robots. Discrete and Continuous Dynamical Systems B, 18, 1189-1215 (2013).

2. Amestoy, P.R., Duff, I.S., Koster, J., LÉxcellent, J.Y.: A fully asynchronous multifrontal solver using distributed dynamic scheduling. SIAM Journal of Matrix Analysis and Applications, 23(1):15-41, (2001).

3. Becker, L.E., Koehler, S.A., Stone, H.A. On self-propulsion of micro-machines at low Reynolds number: Purcell's three-link swimmer. J. Fluid Mech., (2003).

4. Bonnans, F., Martinon, P., Grélard, V.. Bocop - A collection of examples. Technical report RR-8053, INRIA, (2012=.

5. Coron, J.M.: Control and Nonlinearity. Mathematical Surveys and Monographs, Vol. 136, American Mathematical Society, Providence, (2007).

6. Friedrich, B. M., Riedel-Kruse, I. H., Howard, J., Jülicher, F.: High-precision tracking of sperm swimming fine structure provides strong test of resistive force theory.The Journal of Experiment Biology, 213, 1226-1234 (2010).

7. Gebremedhin, A., Pothen, A., Walther, A.: Exploiting sparsity in jacobian computation via coloring and automatic differentiation: a case study in a simulated moving bed process. In: C. Bischof et al, editor, Lecture Notes in Computational Science and Engineering 64: 339349. Springer, 2008. Proceedings of the Fifth International Conference on Automatic Differentiation (AD2008).

8. Happel, J., Brenner, H.: Low Reynolds number hydrodynamics with special applications to particulate media. (Prentice-Hall, Englewood Cliffs, N.J., 1965).

9. Jurdjevic, V. Geometric control theory. Cambridge University Press., (1997).

10. Purcell, E.M. Life at low Reynolds number. American Journel of Physics, 45:3-11, (1977).

11. Riedel-Kruse, I. H., Hilfinger, A., Howard, J., Jülicher, F.: How molecular motors shape the flagellar beat. HFSP, (2007).

12. Trelat, E.. Contrôle optimal : théorie and applications. Vuibert, Collection Mathématiques Concrètes, (2005).

13. Wächter, A. Biegler, L.T.: On the implementation of a primal-dual interior point filter line search algorithm for large-scale nonlinear programming. Mathematical Programming, 106(1), 25-57, (2006).

14. Walther, A., Griewank, A. Getting started with Adol-c. In: U. Naumann and O. Schenk, editors, Combinatorial Scientific Computing. Chapman-Hall CRC Computational Science, (2012).

15. Broy, M.: Software engineering - from auxiliary to key technologies. In: Broy, M., Dener, E. (eds.) Software Pioneers, pp. 10-13. Springer, Heidelberg (2002)

16. Dod, J.: Effective substances. In: The Dictionary of Substances and Their Effects. Royal Society of Chemistry (1999) Available via DIALOG. http://www.rsc.org/dose/title of subordinate document. Cited 15 Jan 1999

17. Geddes, K.O., Czapor, S.R., Labahn, G.: Algorithms for Computer Algebra. Kluwer, Boston (1992)

18. Hamburger, C.: Quasimonotonicity, regularity and duality for nonlinear systems of partial differential equations. Ann. Mat. Pura. Appl. 169, 321-354 (1995)

19. Slifka, M.K., Whitton, J.L.: Clinical implications of dysregulated cytokine production. J. Mol. Med. (2000) doi: 10.1007/s001090000086

20. J. Dod, in The Dictionary of Substances and Their Effects, Royal Society of Chemistry. (Available via DIALOG, 1999), http://www.rsc.org/dose/title of subordinate document. Cited 15 Jan 1999

21. H. Ibach, H. Lüth, Solid-State Physics, 2nd edn. (Springer, New York, 1996), pp. 45-56

22. S. Preuss, A. Demchuk Jr., M. Stuke, Appl. Phys. A 61

23. M.K. Slifka, J.L. Whitton, J. Mol. Med., doi: 10.1007/s001090000086 
24. S.E. Smith, in Neuromuscular Junction, ed. by E. Zaimis. Handbook of Experimental Pharmacology, vol 42 (Springer, Heidelberg, 1976), p. 593

25. Calfee, R. C., \& Valencia, R. R. (1991). APA guide to preparing manuscripts for journal publication. Washington, DC: American Psychological Association.

26. Dod, J. (1999). Effective substances. In: The dictionary of substances and their effects. Royal Society of Chemistry. Available via DIALOG. http://www.rsc.org/dose/Effective substances. Cited 15 Jan 1999.

27. Harris, M., Karper, E., Stacks, G., Hoffman, D., DeNiro, R., Cruz, P., et al. (2001). Writing labs and the Hollywood connection. J Film Writing, 44(3), 213-245.

28. O’Neil, J. M., \& Egan, J. (1992). Men's and women's gender role journeys: Metaphor for healing, transition, and transformation. In B. R. Wainrig (Ed.), Gender issues across the life cycle (pp. 107-123). New York: Springer.

29. Kreger, M., Brindis, C.D., Manuel, D.M., Sassoubre, L. (2007). Lessons learned in systems change initiatives: benchmarks and indicators. American Journal of Community Psychology, doi: 10.1007/s10464-007-9108-14.

30. Alber John, Daniel C. O'Connell, and Sabine Kowal. 2002. Personal perspective in TV interviews. Pragmatics 12:257-271

31. Cameron, Deborah. 1997. Theoretical debates in feminist linguistics: Questions of sex and gender. In Gender and discourse, ed. Ruth Wodak, 99-119. London: Sage Publications.

32. Cameron, Deborah. 1985. Feminism and linguistic theory. New York: St. Martin's Press.

33. Dod, Jake. 1999. Effective substances. In: The dictionary of substances and their effects. Royal Society of Chemistry. Available via DIALOG. http://www.rsc.org/dose/title of subordinate document. Cited 15 Jan 1999

34. Suleiman, Camelia, Daniel C. OConnell, and Sabine Kowal. 2002. 'If you and I, if we, in this later day, lose that sacred fire...': Perspective in political interviews. Journal of Psycholinguistic Research. doi: 10.1023/A:1015592129296.

35. Brown B, Aaron M (2001) The politics of nature. In: Smith J (ed) The rise of modern genomics, 3rd edn. Wiley, New York

36. Dod J (1999) Effective Substances. In: The dictionary of substances and their effects. Royal Society of Chemistry. Available via DIALOG. http://www.rsc.org/dose/title of subordinate document. Cited 15 Jan 1999

37. Slifka MK, Whitton JL (2000) Clinical implications of dysregulated cytokine production. J Mol Med, doi: 10.1007/s001090000086

38. Smith J, Jones M Jr, Houghton L et al (1999) Future of health insurance. N Engl J Med 965:325-329

39. South J, Blass B (2001) The future of modern genomics. Blackwell, London 



\section{Index}

BOCOP, 17

Controllability, 12

Curvature approximation, 8

geometric control, 13

Lie algebra, 13

minimum time optimal control problem, 16
$\mathrm{N}$-link swimmer, 2

Purcell stroke, 19

Purcell's swimmer, 17

Resistive Force Theory, 3

sperm cell, 9 